\title{
A COMPARATIVE ANALYSIS OF ALTERNATIVE UNIVARIATE TIME SERIES MODELS IN FORECASTING TURKISH INFLATION
}

\author{
A. Nazif Çatık ${ }^{1}$, Mehmet Karaçuka ${ }^{2}$ \\ Department of Economics, Ege University, 35040 Bornova / Izmir, Turkey \\ E-mails: ${ }^{1}$ a.nazif.catik@ege.edu.tr (corresponding author); \\ 2mehmet.karacuka@ege.edu.tr \\ Received 26 January 2011; accepted 28 April 2011
}

\begin{abstract}
This paper analyses inflation forecasting power of artificial neural networks with alternative univariate time series models for Turkey. The forecasting accuracy of the models is compared in terms of both static and dynamic forecasts for the period between 1982:1 and 2009:12. We find that at earlier forecast horizons conventional models, especially ARFIMA and ARIMA, provide better one-step ahead forecasting performance. However, unobserved components model turns out to be the best performer in terms of dynamic forecasts. The superiority of the unobserved components model suggests that inflation in Turkey has time varying pattern and conventional models are not able to track underlying trend of inflation in the long run.
\end{abstract}

Keywords: inflation forecasting, neural networks, unobserved components model.

Reference to this paper should be made as follows: Çatık, A. N.; Karaçuka, M. 2012. A comparative analysis of alternative univariate time series models in forecasting Turkish inflation, Journal of Business Economics and Management 13(2): 275-293.

JEL Classification: C45, C53, E31, E37.

\section{Introduction}

The stability of prices, as an important indicator of overall economic performance is one of the main objectives of monetary policy. Turkish economy has a long history of instable macroeconomic performance, especially the persistence of high inflation rates, which lasted for almost five decades. During this long period, many stability programs, mostly backed by the IMF, could not be implemented thoroughly, and failed to achieve the desired outcome, economic and price stability. After the collapse of IMF-supported program in February 2001, Turkey faced a financial turmoil, followed by a destructive overall economic crisis. Consuming all other options, Central Bank of The Republic of Turkey (CBRT) announced to implement new policies that focus on monetary targeting and at the same time on the future inflation. The meaning of this policy was an "implicit" inflation targeting, and in 2005 the CBRT started to implement "explicit" inflation targeting policy, assuming that the conditions of success were available. 
Although, inflation targeting was started on a trial and error basis of the central banks in New Zealand, Canada, England and Sweden with little academic and theoretical background, its popularity has let a growing academic effort to analyze the effectiveness and overall macroeconomic effects of these policies. The main characteristics of inflation targeting policy are described by Mishkin (2000) as; the public announcement of a well defined numerical target for inflation; a commitment to price stability as the primary goal of monetary policy; an information inclusive strategy for deciding the setting of policy instruments; increased transparency of the monetary policy strategy; and increased accountability of the central bank for attaining its inflation objectives.

While predicting future values of price changes accurately is essential for inflation targeting policy, and also for the credibility of monetary policy, there is no consensus on a superior methodology. The reason is that the behavior of price movements is complex, volatile and nonlinear in a wide range. The empirical literature on forecasting inflation rates suggest that there is no single methodology that can outperform others in a case independent context. The recent advance in the applicability of nonlinear models has changed the path of the discussion mainly on the comparison of linear and nonlinear models to describe the behavior of time series. Among others, artificial neural network models (ANNs) have emerged as a powerful statistical modeling technique for nonlinear modeling of time series and applied into various case studies (Gonzalez 2000; Tkacz 2001; Zhang et al. 1998). Although these models have numerous advantages such as letting more general and flexible formations that can approximate any continuous measurable function with arbitrarily desired accuracy level (Cybenko 1989; Hornik et al. 1989; Hornik 1993; Koutroumanidisa et al. 2011), the theoretical superiority of ANNs to the linear models is not yet to be justified in forecasting performances (Moshiri, Cameron 2000; Clements et al. 2004) $)^{1}$.

Even though a long historical experience with high inflation levels in Turkey, the topic seems to be neglected given its impact on social and economic structure until recently. Domaç (2004) estimates various univariate and multivariate linear time series models to understand dynamics of inflation in Turkey using monthly data covering the period 1990-01-2002-12. In this study he estimates mark-up models, monetary models, and the Phillips curve along with the simple univariate autoregressive model. He finds that the mark up models have the best in-sample performance, whereas the Phillips curve and the money gap models turn out to be better in terms of out-of-sample forecasting accuracy. Önder (2004) compares the forecasting performance of the Phillips curve with autoregressive intregrated moving average (ARIMA), vector autoregression, vector error correction and a random walk model in Turkey for the period between 1987 and 2001. She finds that Phillips curve provides better forecasting accuracy than the other alternative models.

In this study we evaluate inflation forecasting accuracy of alternative univariate time series models, i.e. random walk, ARIMA (Autoregressive Integrated Moving Average), ARFIMA (Autoregressive Fractionally Integrated Moving Average), FIGARCH (Frac-

\footnotetext{
${ }^{1}$ Some studies find evidence in favour of neural network models (see for example Binner et al. 2004; Nakamura 2005; Panda, Narasimhan 2007) whereas the others not (Faraway, Chatfield 1998; Stock, Watson 1998; Heravi et al. 2004).
} 
tionally Integrated GARCH), unobserved components models (UCM) and ANNs. We find that when the static forecasts are taken into consideration, ARFIMA and ARIMA models produce better forecasts than UCM and ANN models at earlier forecast horizons. However, in terms of dynamic forecasts UCM and ANN models turn out to have better forecasting accuracy than the other models.

The rest of the paper is organized as follows. First, the basic descriptive properties of the inflation data are presented in Section 2, followed by a brief description of the methodologies used in the study in Section 3. Empirical findings of the study are presented in Section 4. Finally some conclusions and policy proposals are put forward in the last section.

\section{Data}

This paper uses monthly seasonally adjusted Turkish CPI (Consumer Price Indices) data collected from International Financial Statistics (IFS) online database covering the period 1982:01-2009:12 . Inflation $\left(\pi_{t}\right)$ is defined as month on month changes in the natural $\log$ of CPI (see Figure 1).

Before proceeding, we summarize distribution and time series properties of the inflation (see Table A1 and Figure A1). Skewness and kurtosis coefficients of inflation are higher than that of the standard normal distribution and Jarque-Bera statistic, which has the critical value of 5.99 at $5 \%$ significance level, indicates that $\pi_{t}$ is not normally distributed. The same descriptive statistics computed for the period after 2003 suggest that inflation follows relatively stable pattern after the implementation of inflation targeting strategy. Density function and Quantile-Quantile (QQ) plot are further supportive of this fact. ADF unit root test implies the stationarity of inflation. Partial autocorrelation function indicates existence of positive autocorrelation especially at first, fourth, fifth and twelfth lags. The estimated spectral density function which is defined as a smoothed function of the sample autocorrelations shows that $\pi_{t}$ is stationary, but autocorrelated.

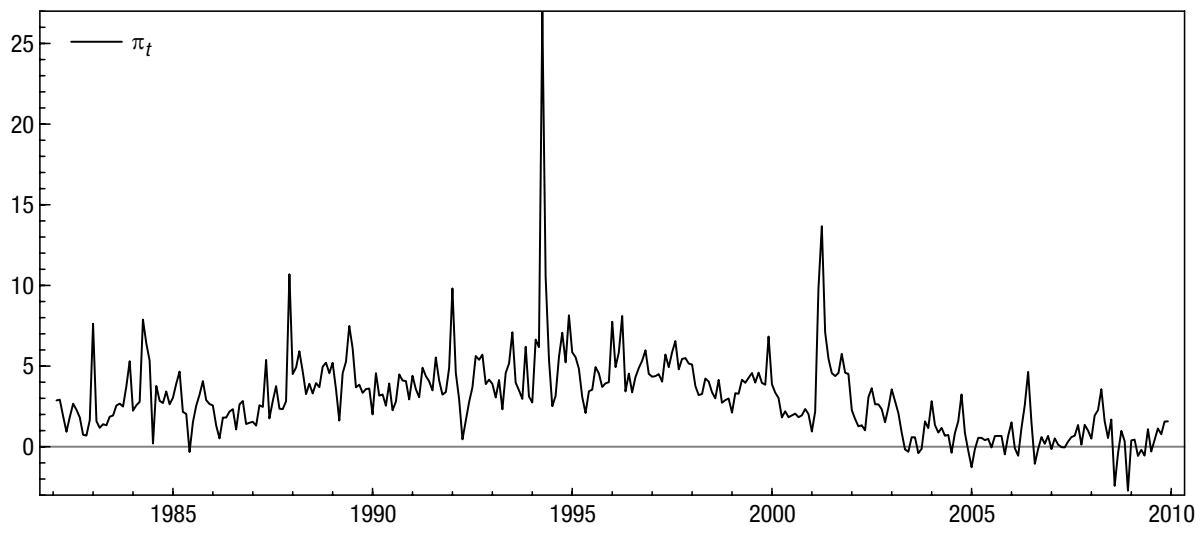

Fig. 1. CPI inflation in Turkey: 1982:02-2009:12

\footnotetext{
${ }^{2}$ Series available on IFS database is not originally seasonally adjusted. In order to obtain adjusted CPI series we employ Tramo-Seats method.
} 


\section{Forecasting models}

\subsection{Artificial neural network models}

In recent years artificial neural network models have become a popular tool in the forecasts of financial and macroeconomic time series ${ }^{3}$. ANNs are nonlinear models that can be used to explain relations which may be difficult to model with linear constant parameter models (Gonzalez 2000). It contains interconnected group of artificial neurons just as the structure of the human brain that helps to learn the pattern of the data.

In contrast to conventional model-based methodologies, ANNs do not require a priori assumptions about the relationship. Due to its nonlinear structure, they can be approximated into any continuous functions and they are known as universal approximation (Hornik et al. 1989; Hill et al. 1994). In spite of these advantages, forecasting with ANNs is not an easy task since there is no consensus about determining the parameters of the models, such as architecture, size of training and validation sets, and the type of activation function. In most studies those factors, playing vital role in the success of ANNs, are rather determined through successive experiments (Zhang et al. 1998; Panda, Narasimhan 2007).

As far as their architecture is concerned, ANNs can be divided into two categories: Feedback (recurrent) and feed-forward neural networks. Feed-forward ANNs work only one direction from inputs to output, whereas feedback ANNs are also able to move into both directions. Feedback ANNs are not suitable for time series forecasting, since they require twice times higher number of parameters than the feed-forward ANNs. Hence we use fully connected feed-forward network with one hidden layer which is the most popular ANN models for time series forecasting applications (Tkacz 2001; Nakamura 2005). ANN model used in this study can be formulated as ${ }^{4}$

$$
\hat{\pi}_{t+1}=\delta_{0}+\sum_{j=1}^{r} \delta_{j} f\left(\sum_{i=0}^{p} \varphi_{i k} \pi_{t-i}+\varphi_{o k}\right)+\varepsilon_{t},
$$

where $\delta_{0}$ is a bias vector $\left\{\delta_{k}, k=0,1, \cdots, r\right\}$ is a vector of weights from the hidden to output nodes and $\left\{\varphi_{i k}, i=0,1, \cdots, p ; k=1,2, \cdots, \ell\right\}$ are weights from the input to hidden nodes, in our case they are parameters of current and lagged values of inflation. $p$ is the number of input nodes, $r$ is the number of hidden nodes, $f$ is a nonlinear activation function by which information transmitted to the next neuron. Given the availability of various activation functions, such as linear, hyperbolic tangent and logistic, we used the most preferred the logistic sigmoidal function $f(x)=1 / 1+e^{-\lambda}$, following the previous literature (Tkacz 2001; Panda, Narasimhan 2007; Binner et al. 2004). In this framework ANN model in (1) can be conceived as a nonlinear autoregressive (AR) model.

\footnotetext{
${ }^{3}$ See Zhang et al. (1998) for detailed literature survey on forecasting with ANN.

${ }^{4}$ See Figure A2 in Appendix for the general architecture of the feed-forward neural network model.
} 


\subsection{Linear time series models}

In order to evaluate forecasting performance of ANNs, we consider three univariate time series models. The first model is the following first order random walk (RW) without drift model of inflation,

$$
\pi_{t}=\rho_{1} \pi_{t-1}+u_{t},
$$

where $\rho_{1}$ is autoregressive parameter to be estimated and $u_{t}$ is i.i.d. error term. By adding a constant and moving average term to RW model, Equation (2) can be converted into $\operatorname{ARIMA}(p, d, q)$ model as follows:

$$
\pi_{t}=c_{0}+\sum_{j=1}^{p} \phi_{j} \pi_{t-i}+\sum_{j=1}^{q} \theta_{j} \varepsilon_{t-i}+u_{t} .
$$

Where, $p, d$ and $q$ are integers referring the order of the autoregressive, integrated and moving average parts respectively. Time series properties of inflation in the previous section suggest that inflation data may also exhibit long-memory properties: autocorrelation and spectral density functions decay slowly but there is no indication for nonstationarity. Therefore, in addition to $\operatorname{ARMA}(p, d, q)$ model above we also use the long-memory ARFIMA model in forecasting inflation. The $\operatorname{ARFIMA}(p, d, q)$ model for $\pi_{t}$ is written as,

$$
\Phi(L)(1-L)^{d}\left(\pi_{t}-\mu_{t}\right)=\Theta(L) \varepsilon_{t}, t=1, \ldots, T .
$$

Where $\Phi(L)$ and $\Theta(L)$ refer to autoregressive and moving average polynomials respectively. $(1-L)^{d}$ is the fractional difference parameter. The stationarity of $\pi_{t}$ depends on the value of $d$. ARFIMA(p,d,q) process is said to be covariance stationary if $d<0.5$. In that case autocovariance function decays hyperbolically. The process is long memory stationary if $0<d<0.5$. For $-0.5<d<0$ the process is known as intermediate memory, and when $d \geq 0.5$ the process is said to be nonstationary.

The descriptive analysis of $\pi_{t}$ also implies that the distribution of inflation shows nongaussian behavior, it is right skewed and has fat tails. In order to account for this type of asymmetry, one solution is modeling $\pi_{t}$ with GARCH-type models with a skewed density function. For this purpose, we employed Fractionally Integrated GARCH (FIGARCH) model introduced by Baillie et al. (1996). This model allows us to analyze the dynamic adjustments of both the conditional mean and the conditional variance of inflation over time. Following Lambert and Laurent (2000) non-normality of inflation is considered by employing skewed-student density function instead of normal density in the maximum likelihood estimates.

The mean equation of the FIGARCH is identical to ARIMA specification in (3). Additionally, the conditional variance equation of the model is formulated as follows,

$$
1-\beta(L) h_{t}=w+\left[(1-\beta(L))-(1-\alpha(L))(1-L)^{d_{v}}\right] \varepsilon_{t}^{2},
$$

where $d_{v}$ is a fractional parameter of the variance equation ranging from 0 to 1 and $\alpha$ and $\beta$ are the respective $\mathrm{ARCH}$ and $\mathrm{GARCH}$ parameters satisfying $\alpha, \beta<1$ condition. 
The functional form of FIGARCH model presented in (3) and (5) is very flexible ${ }^{5}$. For $d_{v}=0$ the specification collapses into conventional GARCH model, whereas for $d_{v}=$ 1 model becomes an Integrated GARCH. The process is reduces to ARFIMA model when $h_{t}$ a constant.

In addition to autoregressive time series models, we also consider a univariate unobserved components model (UCM) of inflation, also known as local level model, having the following state space form (Harvey 1989; Commandeur, Koopman 2007),

$$
\begin{array}{rlrl}
\pi_{t} & =\mu_{t}+\varepsilon_{t}, & & \varepsilon_{t} \sim \operatorname{NID}\left(0, \sigma_{\varepsilon}^{2}\right), \\
\mu_{t} & =\mu_{t-1}+\eta_{t}, & \eta_{t} \sim \operatorname{NID}\left(0, \sigma_{\eta}^{2}\right), \quad t=1, \ldots, n .
\end{array}
$$

Where $\mu_{t}$ is time varying unobserved level, $\varepsilon_{t}$ and $\eta_{t}$ are i.i.d. disturbances of measurement and transition equations. This model allows us to decompose inflation in two parts as level $\mu_{t}$ long run component and irregular components $\varepsilon_{t}$ and $\eta_{t}$. The second equation in (6) known as transition equation indicates that unobserved level of inflation is time varying and assumed to follow first order random walk process over time. The ratio between variances of the level and irregular components $\sigma_{\eta}^{2} / \sigma_{\varepsilon}^{2}$ is called signal to noise ratio. After maximum likelihood estimation of the parameter level $\mu_{t}$ is obtained through a Kalman filter. This process is also known as smoothing or signal extraction.

\section{Empirical results}

\subsection{Model estimations}

In this section, the models defined in the previous section are estimated. First we start from the neural network model. The estimation of the ANNs includes two steps. Training stage involves an iterative procedure where optimum hidden node and output node weights are adjusted by means of neural network algorithm. After the training, the model can be used to produce fitted or forecasted values. The sample period up to 2007:12 is accepted as the training set for ANN model, the remaining 24 observations are reserved to testing set.

We use backpropagation techniques with an adaptive learning rate algorithm to train the model to specified level of convergence. As a standard procedure, $\pi_{t}$ is mapped linearly into the range 0 and 1 to adjust for the logistic activation function ${ }^{6}$. Our model is trained until the mean square error, the mean squared error between the actual and the fitted values of the output of the ANN, lies below the convergence criterion. As previously stated, determining the number of input and output nodes of the network is very important to produce good forecasts. We conduct estimations to find out whether a change in the number of input and output nodes makes any effect on the training performance. For this purpose RMSE values obtained from the training set of the models that contain different combinations of input and hidden nodes are reported in Table 1.

\footnotetext{
${ }^{5}$ For detailed information on the estimation of FIGARCH model see Chung (1999).

${ }^{6}$ In order to obtain the data on normalized inflation $\pi_{t}^{\text {norm }}$, we use the following conventional formula (Mehrotra et al. 1996; Panda, Narasimhan 2007) $\pi_{t}^{\text {norm }}=\left(\pi_{t}-\pi_{t}^{\min }\right) /\left(\pi_{t}^{\max }-\pi_{t}^{\min }\right) \cdot \pi_{t}^{\min }$ and $\pi_{t}^{\max }$ denote minimum and maximum values of inflation respectively.
} 
RMSE values decrease with the increase in the number of input and hidden nodes used in the estimation. RMSE is minimized when the model is estimated with 10 and 11 input nodes. Hence we retain the ANN model with 11 and 4 hidden nodes as a best performer in training to use for the out of sample forecast comparison.

Table 1. Training performance of the alternative ANN specifications

\begin{tabular}{|c|c|c|c|c|c|}
\hline $\begin{array}{l}\text { Number of } \\
\text { Input Nodes }\end{array}$ & $\begin{array}{l}\text { Number of } \\
\text { Hidden Nodes }\end{array}$ & RMSE & $\begin{array}{l}\text { Number of } \\
\text { Input Nodes }\end{array}$ & $\begin{array}{l}\text { Number of } \\
\text { Hidden Nodes }\end{array}$ & RMSE \\
\hline 1 & 1 & 0.0833 & 7 & 1 & 0.0430 \\
\hline 1 & 2 & 0.0641 & 7 & 2 & 0.0425 \\
\hline 1 & 3 & 0.0649 & 7 & 3 & 0.0424 \\
\hline 1 & 4 & 0.0649 & 7 & 4 & 0.0398 \\
\hline 2 & 1 & 0.0441 & 8 & 1 & 0.0429 \\
\hline 2 & 2 & 0.0436 & 8 & 2 & 0.0424 \\
\hline 2 & 3 & 0.0447 & 8 & 3 & 0.0411 \\
\hline \multirow[t]{2}{*}{2} & 4 & 0.0447 & 8 & 4 & 0.0396 \\
\hline & & 0.0443 & & & 0.0415 \\
\hline 3 & 1 & 0.0559 & 9 & 1 & 0.0427 \\
\hline 3 & 2 & 0.0544 & 9 & 2 & 0.0424 \\
\hline 3 & 3 & 0.0541 & 9 & 3 & 0.0412 \\
\hline 3 & 4 & 0.0447 & 9 & 4 & 0.0412 \\
\hline 4 & 1 & 0.0544 & 10 & 1 & 0.0426 \\
\hline 4 & 2 & 0.0529 & 10 & 2 & 0.0412 \\
\hline 4 & 3 & 0.0447 & 10 & 3 & 0.0400 \\
\hline 4 & 4 & 0.0447 & 10 & 4 & 0.0387 \\
\hline 5 & 1 & 0.0447 & 11 & 1 & 0.0425 \\
\hline 5 & 2 & 0.0447 & 11 & 2 & 0.0424 \\
\hline 5 & 3 & 0.0428 & 11 & 3 & 0.0400 \\
\hline 5 & 4 & 0.0424 & 11 & 4 & 0.0387 \\
\hline 6 & 1 & 0.0429 & 12 & 1 & 0.0436 \\
\hline 6 & 2 & 0.0424 & 12 & 2 & 0.0436 \\
\hline 6 & 3 & 0.0424 & 12 & 3 & 0.0413 \\
\hline 6 & 4 & 0.0388 & 12 & 4 & 0.0412 \\
\hline
\end{tabular}

Note: RMSE calculations are based on the normalized values of the output variable 
After the ANN, we estimate RW and ARIMA models. We do not employ Box and Jenkins (1976) approach in the modeling. From the forecasting point of view it may be disadvantageous to select $p$ and $q$ arbitrarily large. A higher order model will produce lower error give the best fit in sample, but when the model is used for out of sample forecasting purpose, it is likely to produce worse forecast than the lower order model, since the mean square error of the forecasts errors will not affected by only the stationary variance of the model but also by errors arising from the estimation of the parameters of the model (Brockwell, Davis 2002). Therefore we estimate different combinations of ARIMA models with maximum lag order for $p=15$ and $q=2$ to overcome this problem. ARIMA $(8,0,0)$ is found to be the best model selected by Akaike Information Criterion (See Table 2) ${ }^{7}$. The sum of autoregressive parameters is obtained as 0.834 confirming the persistent behavior of inflation over the investigation period. Diagnostic tests reported in Table 2 show that the model suffers from non-normality. They also present some evidence for the first order ARCH effect, indicating that the variance of the error term is not constant over time. However we do not find any sign of autocorrelation in the residuals.

Table 2. Maximum likelihood estimation of ARIMA $(8,0$,0) model: 1982(2)-2009(12)

\begin{tabular}{|c|c|c|}
\hline & Coefficient & S. E. \\
\hline$c_{0}$ & -0.036 & $(0.639)$ \\
\hline$\phi_{1}$ & 0.450 & $(0.054)$ \\
\hline$\phi_{2}$ & 0.092 & $(0.060)$ \\
\hline$\phi_{3}$ & -0.071 & $(0.060)$ \\
\hline$\phi_{4}$ & 0.018 & $(0.059)$ \\
\hline$\phi_{5}$ & 0.177 & $(0.059)$ \\
\hline$\phi_{6}$ & 0.011 & $(0.060)$ \\
\hline$\phi_{7}$ & 0.047 & $(0.060)$ \\
\hline$\phi_{8}$ & 0.110 & $(0.054)$ \\
\hline Log-Likelihood & -692.510 & \\
\hline AIC & 4.194 & \\
\hline$\sigma^{2}$ & 3.667 & \\
\hline \multicolumn{3}{|c|}{ Descriptive Statistics for Residuals } \\
\hline Normality test $\chi^{2}(2)$ & 30.090 & {$[0.0000]$} \\
\hline ARCH test $F(1.324)$ & 3.559 & {$[0.0601]$} \\
\hline Autocorrelation test $\chi^{2}(28)$ & 32.146 & {$[0.2686]$} \\
\hline
\end{tabular}

Note: $* * *$ and $* * *$ indicate significant at 10,5 and $1 \%$ respectively. Numbers in brackets represents the probabilities of residual tests

${ }^{7}$ An automated ARIMA model selection procedure written in RATS is used to obtain AIC for all combinations of ARIMA models. 
The maximum likelihood estimation of the ARFIMA is shown in Table 3. As compared to RW and ARIMA models persistence of inflation remains low, however the model provides similar fit to the data in terms of log-likelihood and Akaike information criteria. The estimated long memory conditional mean parameter $d$ is 0.334 and significantly different from zero. Since the parameter lies in the range $0<d<0.5$, one can say that inflation in Turkey follows a long memory stationary process.

Table 3. Maximum likelihood estimation of ARFIMA (1, d, 0) model: 1982(2)-2009(12)

\begin{tabular}{lcc}
\hline & Coefficient & S.E. \\
\hline$d$ & 0.334 & $(0.059)$ \\
\hline$\Phi_{1}$ & 0.635 & $(0.061)$ \\
\hline Log-Likelihood & -698.424 & \\
\hline AIC & 4.188 & \\
\hline$\sigma^{2}$ & 3.805 & {$[0.0000]$} \\
\hline Descriptive Statistics for Residuals & & {$[0.5150]$} \\
\hline Normality test $\chi^{2}(2)$ & 57.39 & {$[0.1247]$} \\
\hline ARCH test $F(1.331)$ & 0.42479 & \\
\hline Autocorrelation test $\chi^{2}(28)$ & 43.628 & \\
\hline
\end{tabular}

Note: $* * *$ and $* * *$ indicate significant at 10,5 and $1 \%$ respectively. Numbers in brackets represents the probabilities of residual tests

We also estimate FIGARCH model explained above with maximum likelihood method based on Skewed Student distribution, with 2.9092 degrees of freedom. On general, the parameter estimates of the FIGARCH are found to be significant and corroborate the existence of long memory effects as in the ARFIMA model. The inclusion of conditional heteroscedasticity in the residuals improves fit of the model as evidenced by AIC and log-likelihood values (see Table 4). The model also yields a very high and significant fractional difference parameter $d_{v}$ with 0.775 , suggesting that conditional variance of the inflation is characterized by a near integrated GARCH process. The estimated parameter of the skewed student distribution $\psi$ known as the asymmetry parameter within the range of $-1<\psi<1$ is found to be positive and significant supporting right skewed characteristics of the data. Another distribution parameter of the distribution $v$ also confirms heavy-tailed characteristics of the inflation ${ }^{8}$.

Finally we estimate unobserved components model of inflation based on (6). The results of the estimated state vector by maximum likelihood are presented in Table 5. Local level model of inflation passed all diagnostic tests except for normality of the residuals. However cumulative sum of squared residuals (CUSUM) suggest that

\footnotetext{
${ }^{8}$ For detailed information on the functional form of skewed student distribution see Lambert and Laurent (2000).
} 
the model is stable (see Figure A3). The parameter of the unobserved level of inflation is found to be significant, suggesting that underlying level of inflation follows time varying pattern that can be modeled through the first order random walk process (see Figure 2). Estimated state vector shows that inflation has an increasing trend with about $0.94 \%$ on monthly basis.

Table 4. Maximum likelihood estimate of ARMA-FIGARCH model: 1982(2)-2009(12)

\begin{tabular}{lcc}
\hline & Coefficient & S. E. \\
\hline Constant (Mean Eq.) & $3.334^{* * *}$ & $(0.478)$ \\
\hline$\phi_{1}$ & $0.784^{* * *}$ & $(0.051)$ \\
\hline Constant (Variance Eq.) & $4.880^{* * *}$ & $(0.793)$ \\
\hline$d_{v}$ & $0.775^{* * *}$ & $(0.207)$ \\
\hline$\alpha_{1}$ & $0.676^{* * *}$ & $(0.022)$ \\
\hline$\beta_{1}$ & $0.290^{* * *}$ & $(0.040)$ \\
\hline$\psi$ & $0.226^{*}$ & $(0.119)$ \\
\hline$\delta$ & $2.909^{* * *}$ & $(0.352)$ \\
\hline Log-Likelihood & -572.813 & \\
\hline AIC & 3.46754 & \\
\hline Skewness (Y) & 2.89347 & \\
\hline Kurtosis (Y) & 26.18608 & \\
\hline
\end{tabular}

Note: $* * *$ and $* * *$ indicate significant at 10,5 and $1 \%$ respectively. The robust standard errors are given in parentheses. The model is estimated with maximum likelihood method based on Skewed Student distribution, with 2.9092 degrees of freedom

Table 5. Univariate unobserved components model of inflation: 1982(2)-2009(12)

\begin{tabular}{|c|c|c|}
\hline Hyperparameters & Parameter & (q-ratio) \\
\hline Level $\sigma_{\eta}^{2}$ & 0.269 & $(0.086)$ \\
\hline Irregular $\sigma_{\varepsilon}^{2}$ & 3.112 & $(1.000)$ \\
\hline Estimated coefficients of the final state vector & Coefficient & t-values \\
\hline Level $\mu_{t}$ & 0.947 & $(0.019)$ \\
\hline Diagnostic Statistics & Coefficient & p-values \\
\hline$R^{2}$ & 0.342 & \\
\hline Q & 11.362 & $(0.07)$ \\
\hline Normality & 88.460 & $(0.00)$ \\
\hline$H$ & 0.894 & \\
\hline
\end{tabular}

Notes: $R^{2}$ is the coefficient of determination, $\mathrm{Q}$ is Box-Ljung serial correlation statistic, based on the first 12 residual autocorrelations and tested against a chi-square distribution with six degrees of freedom. $H$ is a basic non-parametric test of heteroscedasticity, is a two-sided $\mathrm{F}$ test centered around unity. A low value indicates a decrease in the variance over time. The critical values for this test at the $5 \%$ level are 0.56 and 1.77 

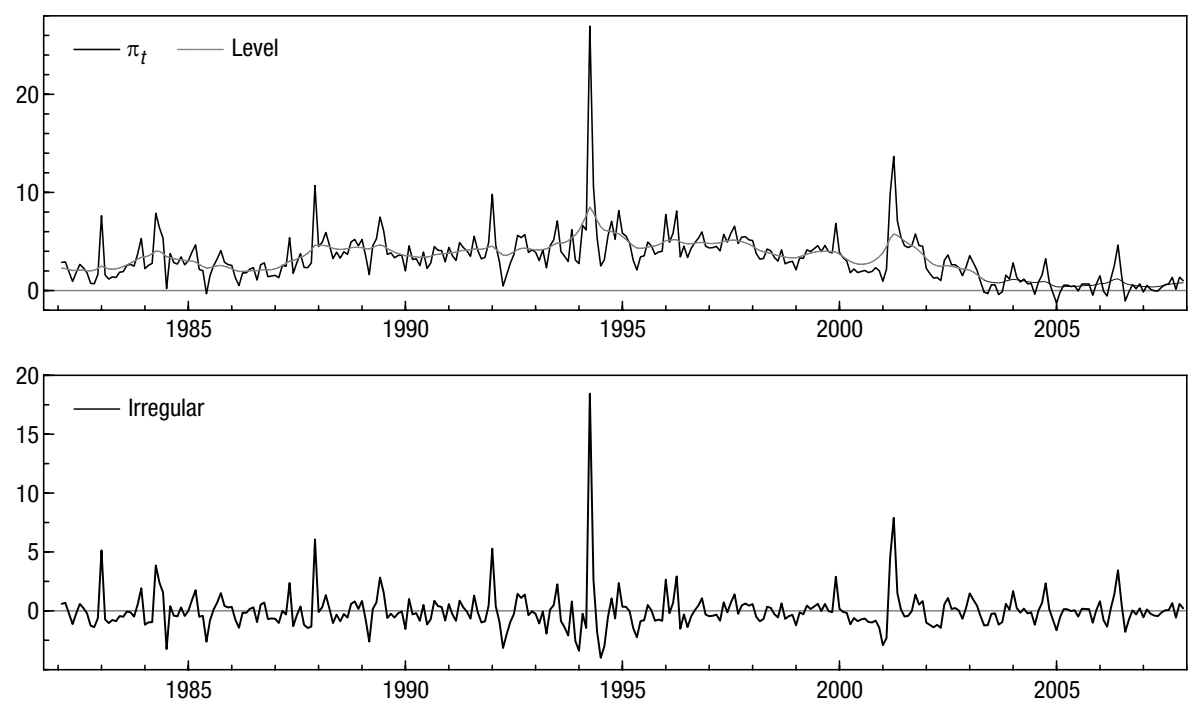

Fig. 2. Level and irregular components of inflation

\subsection{Evaluating forecasting accuracy}

The forecasting accuracy of alternative models is compared in terms of both static and dynamic forecasts. A Static (one-step-ahead) forecast uses the actual values for lagged dependent variables to compute forecasted values of dependent variable. Therefore this technique is more convenient for short term forecasting purpose. On the other hand, a dynamic forecast (multi-step ahead) has ability to produce long term projected values in which forecasted values are generated through an iterative procedure, i.e. forecasted values computed in the earlier periods are used as independent variables (Diebold 2007; Makridakis et al. 1998). Out of sample forecasted values of inflation are computed for each of the models in static and dynamic context. Besides, to account for the impact of change in forecasting path on the accuracy of alternative models we consider different starting points for each forecasting horizon (See Table 6). Hence we allowed each forecasting to end. All estimations are conducted using the sample period up to 2007:12 (training set for ANN model), the remaining 24 observations are reserved to compare out of sample forecasting accuracy (testing set for ANN model).

In order to evaluate the forecasting accuracy of the models, we consider several evaluation criterions. First we compute Mean Absolute Error $(M A E)$ and Root-Mean Squared Error (RMSE) as follows:

$$
\begin{gathered}
R M S E=\sqrt{\sum_{t=T+1}^{T+h} \frac{\left(\hat{\pi}_{t}-\pi_{t}\right)^{2}}{h},} \\
M A E=\sum_{t=T+1}^{T+h} \frac{\left|\hat{\pi}_{t}-\pi_{t}\right|}{h},
\end{gathered}
$$

where $\hat{\pi}_{t}$ and $\pi_{t}$ represents forecasted and actual values of inflation respectively; $h$ 
denotes forecast horizon $h=6,12,18,24$. In addition, scale invariant Theil inequality statistics (Theil) is also computed to evaluate forecasting performance (Pindyck, Rubinfeld 1998).

$$
\text { Theil }=\frac{\sqrt{\frac{1}{h} \sum_{t=1}^{h}\left(\hat{\pi}_{t}-\pi_{t}\right)^{2}}}{\sqrt{\frac{1}{n} \sum_{t=1}^{n} \hat{\pi}_{t}^{2}}+\sqrt{\frac{1}{n} \sum_{t=1}^{n} \pi_{t}^{2}}} .
$$

Theil statictics is always between zero and one, where zero corresponds to perfect fit. Theil inequality statistics can be decomposed into three parts by following two steps. First, mean squared forecast error is divided into three parts:

$$
\sum\left(\hat{\pi}_{t}-\pi_{t}\right) / h=\left(\sum\left(\hat{\pi}_{t} / h-\bar{\pi}\right)\right)^{2}+\left(s_{\hat{\pi}}-s_{\pi}\right)^{2}+2(1-r) s_{\hat{\pi}} s_{\pi},
$$

where $\sum \hat{\pi}_{t} / h, \bar{\pi}, s_{\hat{\pi}}, s_{\pi}$ denote the means and standard deviations of $\hat{\pi}_{t}$ and $\bar{\pi}$, and $r$ represents the correlation between actual and forecasted values. Dividing each part of (8) by the mean squared error itself results in the following decomposition of the Theil inequality statistics,

$$
\frac{\left(\sum\left(\hat{\pi}_{t}-\pi_{t}\right) / h\right)^{2}}{\sum\left(\hat{\pi}_{t}-\pi_{t}\right) / h}+\frac{\left(s_{\hat{\pi}}-s_{\pi}\right)^{2}}{\sum\left(\hat{\pi}_{t}-\pi_{t}\right) / h}+\frac{2(1-r) s_{\hat{\pi}} s_{\pi}}{\sum\left(\hat{\pi}_{t}-\pi_{t}\right) / h}=1 .
$$

The first term is the bias proportion, which shows contribution of the difference in means between the forecasted and actual series to the Theil inequality. The second term is known as the variance proportion, it measures the difference in variance between actual and forecasted values. The last term, the covariance proportion shows remaining unsystematic errors in the forecast. Forecasts are considered as good when the bias proportions are found relatively small compared to covariance proportions. Theil inequality statistics should be mostly explained by the covariance proportions (Pindyck, Rubinfeld 1998).

The criterions described above are computed with different horizons to evaluate outof-sample forecasting accuracy of the alternative models. The findings show that linear models have the lowest RMSE values at shorter forecast horizon, however as forecast horizon gets longer ANN and UCM performs better. Forecasting accuracy statistics are illustrated in Table 6. Random walk without drift model is the worst performer in terms of both static and dynamic forecasts, since it presents highest value of RMSE, MAE and Theil-Inequality statistics at each forecasting horizons. When the one-step ahead forecasts are considered, ARFIMA model turns out be the best performer according to MAE and RMSE criterions (see Figure 3). ARIMA and FIGARCH models are also provide better fit than UCM and ANN at six months. However the performance of UCM has improved considerably with the increase in forecasting horizons, it becomes the second best model after the ARFIMA for the horizons longer than six months. 


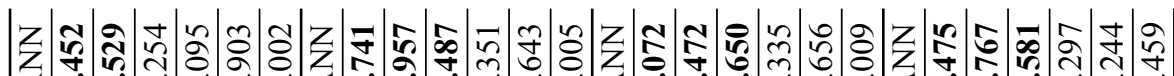

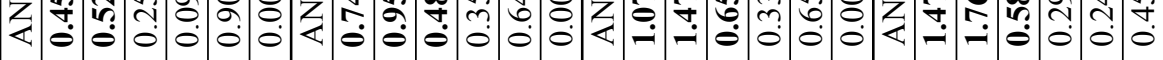

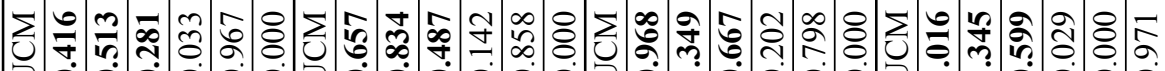
$\checkmark$ ○

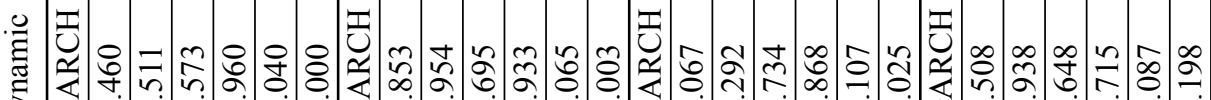
人 。

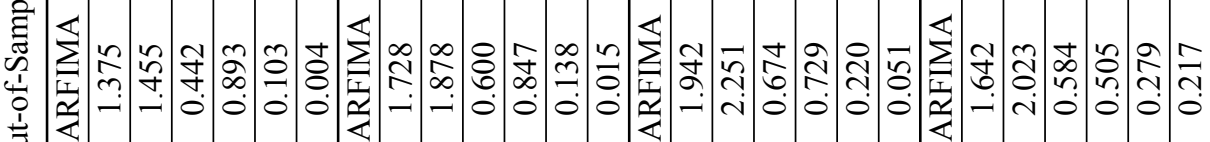
0

\そ)

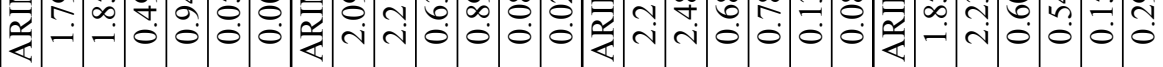
3 m

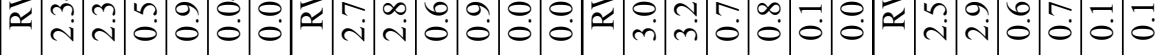

Z

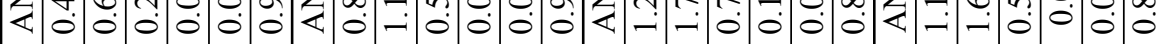

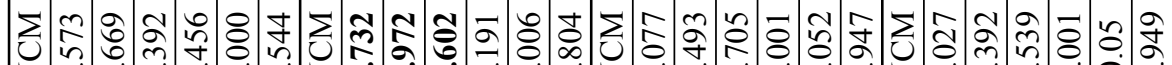
S

己ـ

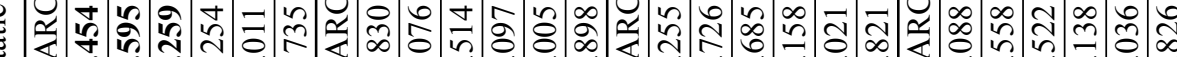
৩ 而

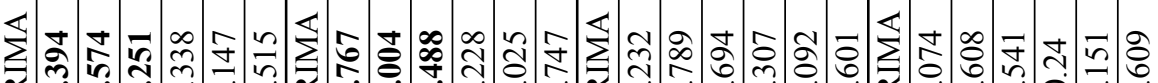
党

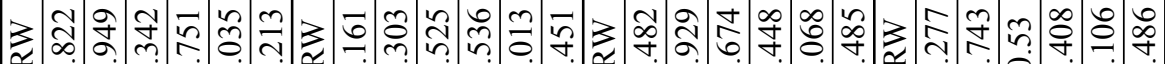

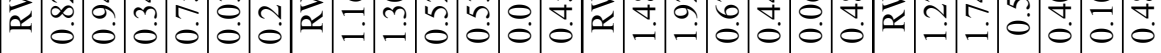

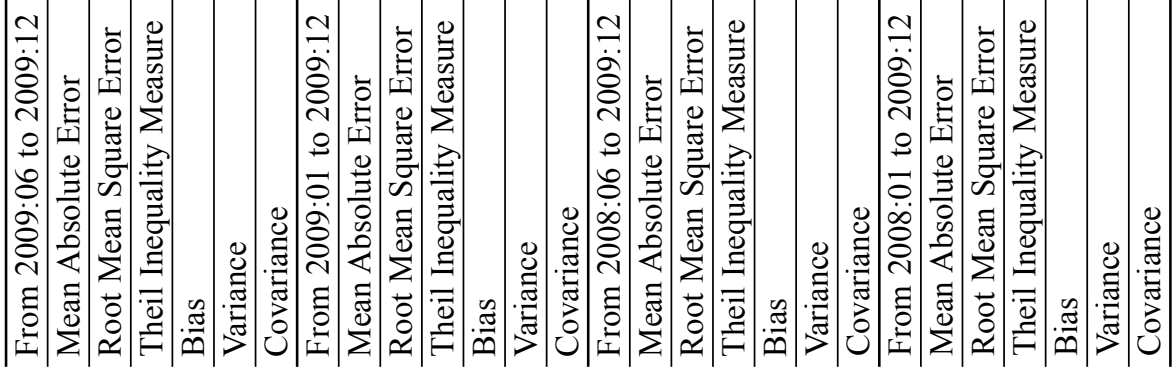



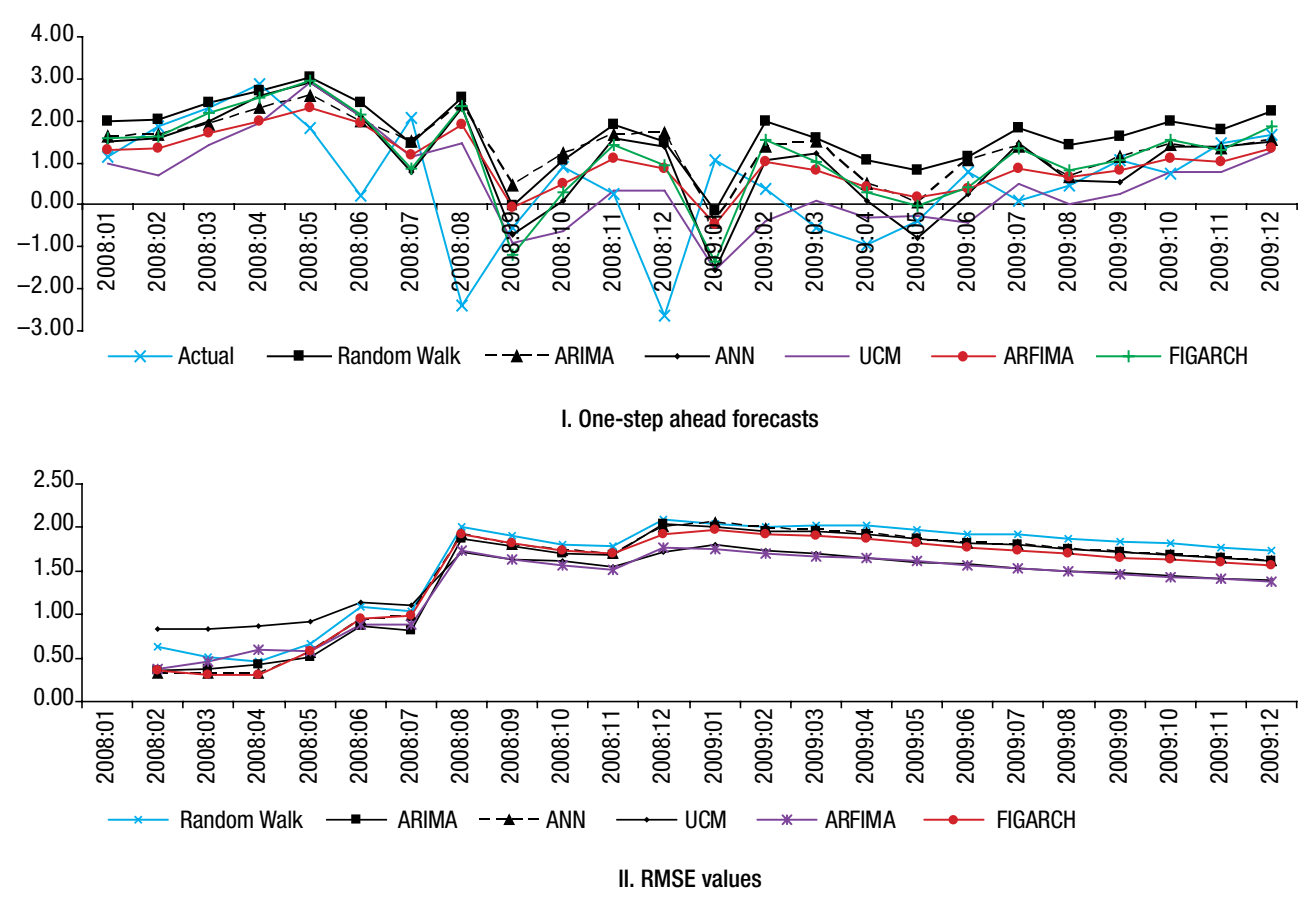

Fig. 3. Out of sample one-step forecasts and RMSE values of the alternative models

The superiority of UCM is more pronounced in terms of dynamic forecasts. The statistics of ANN is very close to that of UCM model up to 18 months. Hence ANN turns out to be the second best model in terms of tracking inflation. In autoregressive models the bias proportion accounts for more than 50 percentage of Theil inequality indicating the presence of systematic error between actual and forecasted values. This also corroborates the evidence on the lower quality of forecasts produced by the other statistics. Overall, the UCM provides superior long-term forecasts compared to the other techniques.

\section{Conclusions}

The stability of prices, as an important indicator of overall economic performance is one of the main objectives of monetary policy. In recent years, inflation targeting, has been implemented by most of the central banks around the world, as a policy tool of maintaining price stability. However, maintaining price stability over a period requires forward-looking approaches due to the lags and dynamic structure of price movements. Therefore, predicting and forecasting inflation has emerged as a crucial factor to be placed in monetary policy decisions for almost every monetary decision making process. In this paper we evaluate inflation forecasting accuracy of alternative time series models. For this purpose, static and dynamic forecasts are produced and the accuracies are compared by RMSE, MAE and Theil inequality measures. Static forecast uses the 
actual values in forecasting, and likely to produce more accurate results for short term forecasting. However, a dynamic forecast employs iterated forecast values and more convenient for long term projections.

We find that at earlier forecast horizons conventional autoregressive models, especially ARIMA and ARFIMA, provide better forecasting performance. However when the horizon gets longer, UCM turns out to be the best performer. The accuracy of ANN model has improved considerably with an increase in the forecast horizon. The superiority of UCM over the alternative models suggests that inflation in Turkey has a time varying pattern and linear models fail to track underlying level of inflation.

These findings indicate that the superiority of the models depends on the forecasting horizon. Inflation targeting policies require long term predictability of price changes, policy makers can consider UCM and ANN models as serious candidates that are able to better track the future values of inflation than the conventional autoregressive models.

\section{References}

Baillie, R. T.; Bollerslev, T.; Mikkelsen, H. O. 1996. Fractionally integrated generalized autoregressive conditional heteroskedasticity, Journal of Econometrics 74: 3-30.

http://dx.doi.org/10.1016/S0304-4076(95)01749-6

Binner, J. M.; Elger, T.; Nilsson, B.; Tepper, J. A. 2004. Tools for non-linear time series forecasting in economics: an empirical comparison of regime switching vector autoregressive models and recurrent neural networks, Advances in Econometrics 19: 71-92.

http://dx.doi.org/10.1016/S0731-9053(04)19003-8

Box, G. E. P.; Jenkins, G. M. 1976. Time Series Analysis: Forecasting and Control. San Francisco: Holden-Day.

Brockwell, P. J.; Davis, R. A. 2002. Introduction to Time Series and Forecasting. Second edition. New York: Springer-Verlag. http://dx.doi.org/10.1007/b97391

Chung, C. F. 1999. Estimating the Fractionally Integrated GARCH Model, Working Paper. National Taiwan University.

Clements, M. P.; Franses, P. H.; Swanson, N. R. 2004. Forecasting economic and financial timeseries with non-linear models, International Journal of Forecasting 20: 169-183.

http://dx.doi.org/10.1016/j.ijforecast.2003.10.004

Commandeur, J. J. F.; Koopman, S. J. 2007. An Introduction to State Space Time Series Analysis. First edition. Oxford: Oxford University Press.

Cybenko, G. 1989. Approximations by superpositions of a sigmoidal function, Mathematics of Control, Signals, and Systems 2: 303-314. http://dx.doi.org/10.1007/BF02551274

Diebold, F. X. 2007. Elements of Forecasting. Fourth edition. Australia: Thomson South-Western.

Domaç, I. 2004. Explaining and Forecasting Inflation in Turkey, World Bank Policy Research Working Paper No. 3287.

Faraway, J.; Chatfield, C. 1998. Time series forecasting with neural networks: a comparative study using airline data, Applied Statistics 47: 231-250. http://dx.doi.org/10.1111/1467-9876.00109

Gonzalez, S. 2000. Neural Networks for Macroeconomic Forecasting: a Complementary Approach to Linear Regression Models, Finance Canada Working Paper No. 2000-07.

Harvey, A. C. 1989. Forecasting Structural Time Series and the Kalman Filter. First edition. Cambridge: Cambridge University Press. 
Heravi, S.; Osborn, D. R.; Birchenhall, C. R. 2004. Linear versus neural network forecasts for European industrial production series, International Journal of Forecasting 20: 435-446.

http://dx.doi.org/10.1016/S0169-2070(03)00062-1

Hill, T.; Marquez, L.; O’Connor, M.; Remus, W. 1994. Artificial neural network models for forecasting and decision making, International Journal of Forecasting 10: 5-15.

http://dx.doi.org/10.1016/0169-2070(94)90045-0

Hornik, K. 1993. Some new results on neural network approximation, Neural Networks 6: 10691072. http://dx.doi.org/10.1016/S0893-6080(09)80018-X

Hornik, K.; Stinnchcombe, M.; White, H. 1989. Multi-layer feed forward networks are universal approximators, Neural Networks 2: 359-366. http://dx.doi.org/10.1016/0893-6080(89)90020-8

Koutroumanidisa, T.; Konstantinos, I.; Zafeirioua, E. 2011. Forecasting bank stock market prices with a Hybrid method: the case of Alpha bank, Journal of Business Economics and Management 12(1): 144-163. http://dx.doi.org/10.3846/16111699.2011.555388

Lambert, P.; Laurent, S. 2000. Modelling Skewness Dynamics in Series of Financial Data. Discussion Paper. Institut de Statistique, Louvain-la-Neuve.

Makridakis, S. G.; Wheelwright, S. C.; Hyndman, R. J. 1998. Forecasting: Methods and Applications. Third edition. New York: John Wiley \& Sons.

Mehrotra, K.; Chilukuri, K. M.; Ranka, S. 1996. Elements of Artificial Neural Networks. Cambridge, MA: MIT Press.

Mishkin, F. S. 2000. Inflation Targeting in Emerging Market Countries, NBER Working Paper No. 7618.

Moshiri, S.; Cameron, N. 2000. Neural networks versus econometric models in inflation forecasting, Journal of Forecasting 19: 201-17.

http://dx.doi.org/10.1002/(SICI)1099-131X(200004)19:3<201::AID-FOR753>3.0.CO;2-4

Nakamura, E. 2005. Inflation forecasting using a neural network, Economic Letters 86: 373-378. http://dx.doi.org/10.1016/j.econlet.2004.09.003

Önder, A. Ö. 2004. Forecasting inflation in emerging markets by using the Phillips curve and alternative time series models, Emerging Markets Finance and Trade 40: 71-82.

Panda, C.; Narasimhan, V. 2007. Forecasting exchange rate better with artificial neural network, Journal of Policy Modeling 29: 227-236. http://dx.doi.org/10.1016/j.jpolmod.2006.01.005

Pindyck, R. S.; Rubinfeld, D. L. 1998. Econometric Models and Economic Forecasts. Fourth edition. New York: McGraw-Hill.

Stock, J.; Watson, M. W. 1998. A Comparison of Linear and Nonlinear Univariate Models for Forecasting Macroeconomic Time Series, NBER Working Paper No. 6607.

Tkacz, G. 2001. Neural network forecasting of Canadian GDP growth, International Journal of Forecasting 17: 57-69. http://dx.doi.org/10.1016/S0169-2070(00)00063-7

Zhang, G. Q.; Patuwo, B. E.; Hu, M. Y. 1998. Forecasting with Artificial neural networks: the State of the art, International Journal of Forecasting 14: 35-62.

http://dx.doi.org/10.1016/S0169-2070(97)00044-7 


\section{APPENDIX}
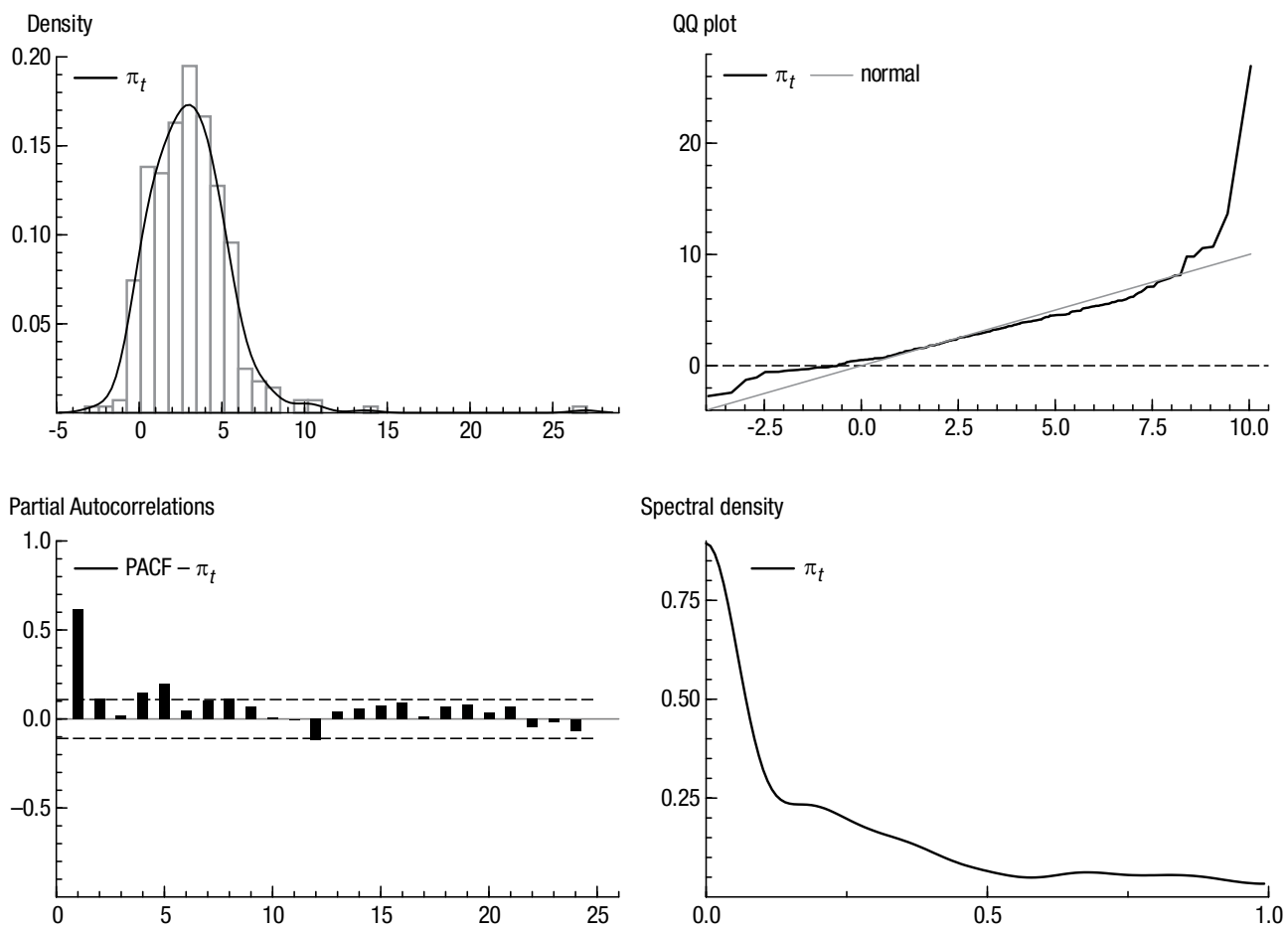

Fig. A1. The distribution and time series properties of $\pi_{t}$

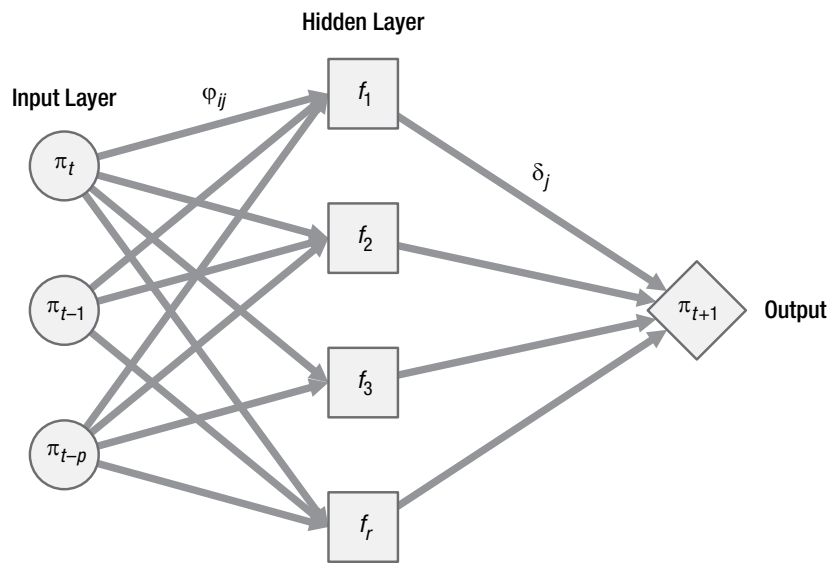

Fig. A2. Feedforward neural network model of inflation 
A. N. Çatık, M. Karaçuka. A comparative analysis of alternative univariate time series models ...
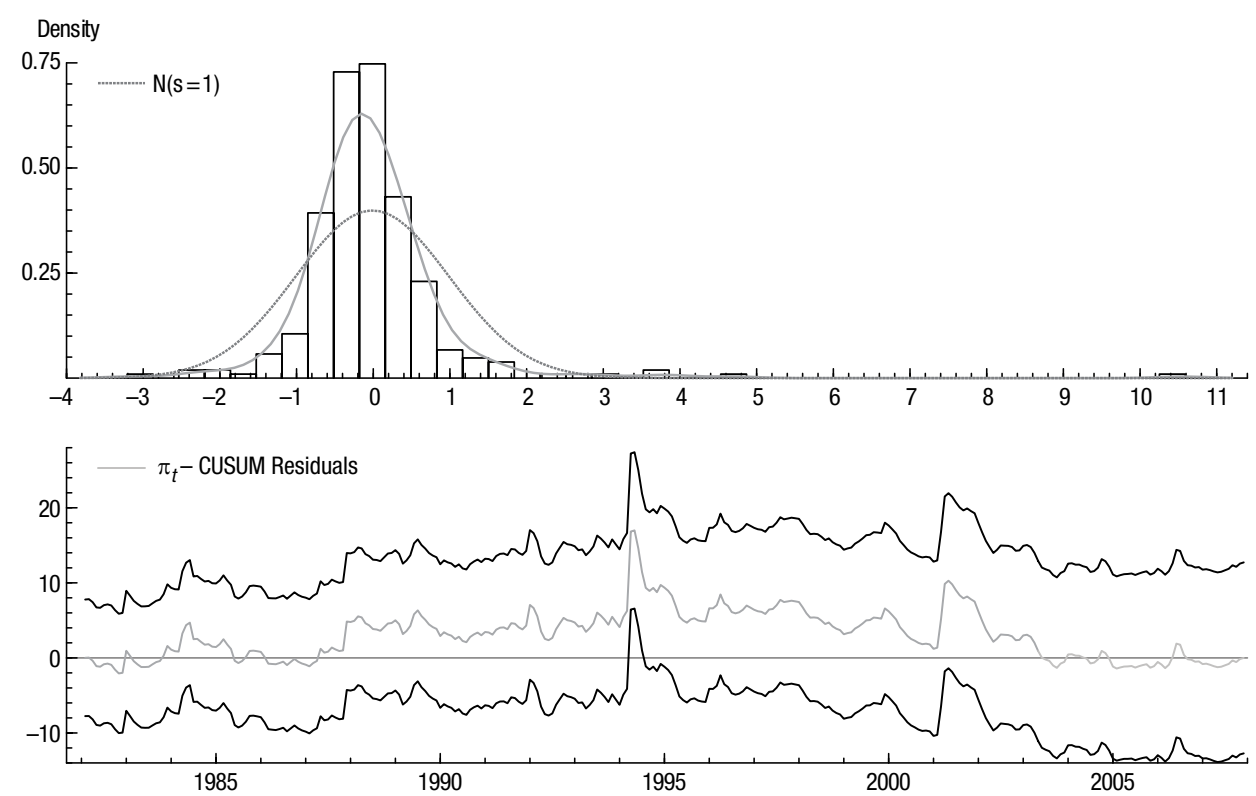

Fig. A3. Residuals of the unobserved components model

Table A1. The descriptive statistics for $\pi_{t}$

\begin{tabular}{lcc}
\hline & Whole Period & After 2003 \\
\hline Mean & 3.048 & 0.703 \\
\hline Median & 2.877 & 0.713 \\
\hline Maximum & 27.004 & 4.316 \\
\hline Minimum & -2.647 & -2.647 \\
\hline Std. Dev. & 2.542 & 1.171 \\
\hline Skewness & 2.893 & 0.273 \\
\hline Kurtosis & 26.186 & 4.236 \\
\hline Jarque-Bera & 7971.366 & 6.391 \\
\hline Probability & 0.000 & 0.041 \\
\hline Sum & 1021.165 & 59.028 \\
\hline Sum Sq. Dev. & 2157.651 & 113.908 \\
\hline Observations & 335 & 84 \\
\hline ADF & -9.680 & -6.159 \\
\hline & $(0.000)$ & $(0.000)$ \\
\hline
\end{tabular}

Note: The critical values for ADF test are $-3.98,-3.42$, and -3.13 at $1 \%, 5 \%$, and $10 \%$ significance level respectively 
A. Nazif ÇATIK is an Assistant Professor at Ege University, Turkey, with Ph.D. His scientific interests are related to the nonlinear analysis of macro data and time series. He has written in cited and indexed journals like Emerging Markets Finance and Trade, Applied Economics Letters, The Journal of Developing Areas, Economics Bulletin, while he has been reviewer in various journals such as METU Development Studies, Journal of Economic Studies. He has published papers in national and international conferences on time series analysis and robust statistics.

Mehmet KARAÇUKA is an Assistant Professor at Ege University, Turkey, with Ph.D. in Economics, and he is teaching Microeconomics and Applied Econometrics. His research interests are Applied Econometric Methods and Industrial Organization. He has written in cited journals like Telecommunications Policy, Applied Economics Letters, The Journal of Developing Areas; while he has been reviewer in different national and international journals such as The Journal of Developing Areas, The Service Industries Journal. 